
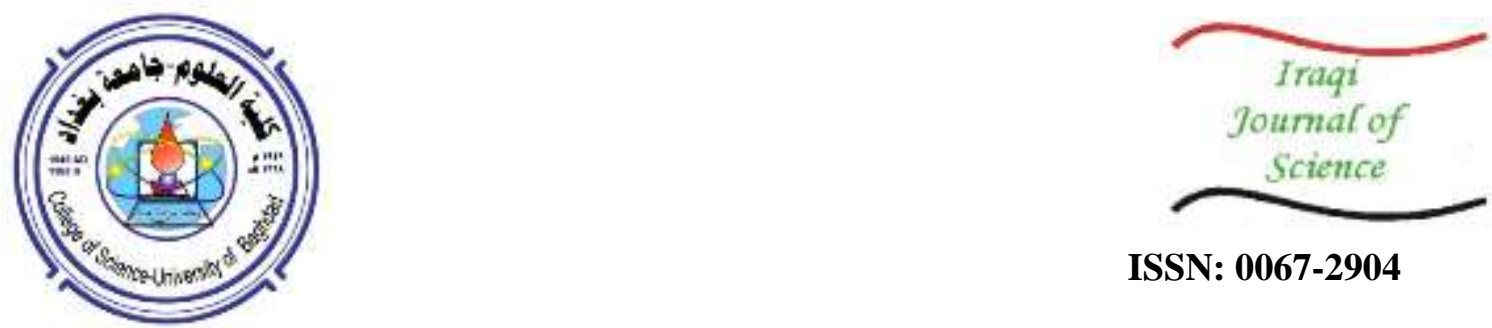

ISSN: 0067-2904

\title{
ON Numerical Blow-Up Solutions of Semilinear Heat Equations
}

\author{
Maan A. Rasheed ${ }^{1}$, Raad A. Hameed ${ }^{2}$, Sameer K. Obeid ${ }^{2}$, Ali F. Jameel ${ }^{3 *}$ \\ ${ }^{1}$ Department of Mathematics, College of Basic Education, Mustansiriyah University, Baghdad, Iraq \\ ${ }^{2}$ Department of Mathematics, College of Education, Tikrit University, Tikrit, Iraq \\ ${ }^{3}$ School of Quantitative Sciences, College of Arts and Sciences,Universiti Utara Malaysia (UUM), Sintok, \\ Kedah, Malaysia
}

Received: 24/9/2019

Accepted: 19/11/2019

\begin{abstract}
This paper is concerned with the numerical blow-up solutions of semi-linear heat equations, where the nonlinear terms are of power type functions, with zero Dirichlet boundary conditions. We use explicit linear and implicit Euler finite difference schemes with a special time-steps formula to compute the blow-up solutions, and to estimate the blow-up times for three numerical experiments. Moreover, we calculate the error bounds and the numerical order of convergence arise from using these methods. Finally, we carry out the numerical simulations to the discrete graphs obtained from using these methods to support the numerical results and to confirm some known blow-up properties for the studied problems.
\end{abstract}

Keywords: Blow-up solution; Semi-linear Heat equation; Dirichlet boundary conditions; Explicit Euler Scheme; Implicit Euler Scheme.

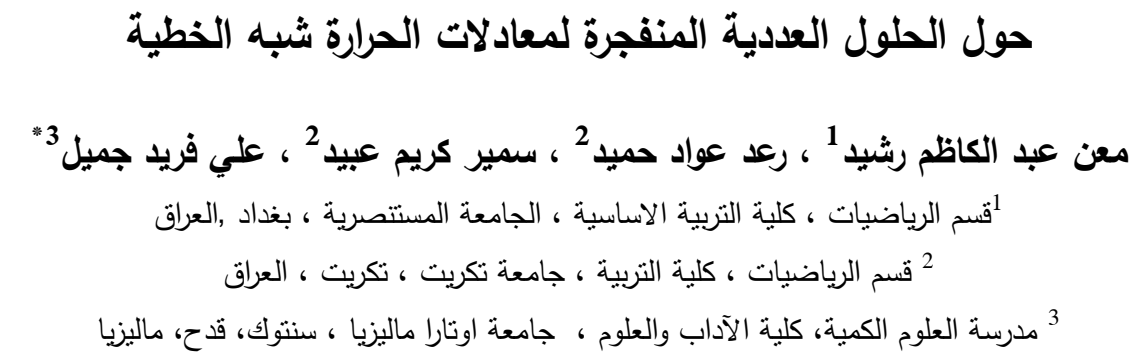

خلاصة

يهنم هذا البحث بالحلول العددية لمعادلات الحرارة شبه الخطية، حيث تكون الحدود الغير خطية دوال من

نوع متعددات الحدود، مع شروط ديرشلت الحدودية المتجانسة. نستخدم طريقتي اوبلر للفروقات المنتهية

الواضحة والضمنية مع صيغة معينة لخطوة الزمن، لحساب الحلول العددية ولتقدير ازمان الانفجار لثناثة

تجارب عددية. علاوة على ذللك، نقوم بحساب حدود الاخطاء ودرجات التقارب العددية الناتجة من استخدام

هذه الطرق. اخيرا، نقوم بتتفيذ عمليات المحاكاة العددية لمنحنيات الدوال المتقطعة التي تم الحصول عليها من لهن

ستخدام هذه الطرق، لاعم النتائج العددية ولتاكيد بعض خصائص التفجيرالمعروفة للمسائل المدروسة.

Email: maan.rasheed.edbs@uomustansiriyah.edu.iq 


\section{INTRODUCTION}

In the field of mathematical modeling, many real life excremental problems can be contracted in the form of partial differential equations in different fields [1]. Some of these equations have semilinear or nonlinear behavior that makes obtaining the exact solution of its governing equation difficult or without exact solution. In that case the alternative way is to use the numerical method to solve these equations such as the Finite Difference Method, which has been used to solve different type of partial differential equations [2-5].

It is well known that semi-linear parabolic equations arise in many physical situations, where the diffusion and source terms have to be modeled. Many of physical situations, including chemical reaction and electrical heating have been presented by Lacey, [6]. In some cases the solution of the semi-linear heat equation cannot be continued globally in time, the so called blow-up phenomena, and that due to the infinite growth of the nonlinear term (source term) describing the evolution process. The phenomenon of blow-up in finite time for semi-linear heat equations has been extensively studied by many authors, and much effort has been made from analytical points of view, see for instance [712]. In this paper, we consider the numerical solutions of the zero Dirichlet problem of a semi linear heat equation:

$$
\left\{\begin{array}{l}
u_{t}=u_{x x}+u^{p}, 0<x<1, t>0 \\
u(x, t)=0, x=0,1 \\
u(x, 0)=u_{0}(x), 0<x<1
\end{array}\right\}
$$

where $p>1 ; u_{0}(x) \in C^{2}(R)$, satisfying $u_{0}(0)=u_{0}(1)=0$. .

For problem (1), it is well known that the local existence of a unique classical solution is guaranteed by standard parabolic theory, see [13]. On the other hand, Friedman and McLeod, [8], have proved that, with a large size initial function, the blow-up in this problem can only occur at a single point.

The study of numerical solutions of time-dependent problems, especially, with blow-up, is at an early stage. However, some authors has considered the numerical solution for some special cases, see [1419].

According to [14], it has been shown that the blow-up solution and numerical blow-up time of the semi discrete problem of (1) converge to the theoretical values as we refine the grids. Moreover, two numerical schemes (explicit and linear implicit Euler) have been used to compute the blow-up solution and estimate the blow-up time for problem (1), where $p=2$, and $u_{0}(x)=20 \sin (\pi x)$, with using the time-step formula:

$k_{n}=\left\{\begin{array}{cc}\min \left(\frac{h^{2}}{2}, \frac{h^{\alpha}}{\left\|U_{h}^{n}\right\|_{\infty}}\right) & \text { for explicit Scheme } \\ \frac{h^{\alpha}}{\left\|U_{h}^{n}\right\|_{\infty}} & \text { for implicit scheme }\end{array}\right\}, n \geq 0, \alpha>0$,

where $h$ is the space-step ; $U_{h}^{n}$ is the vector of numerical solution of the discrete problem. In fact, the reason behind dealing with this type of time-steps rather than fixed time-steps is to ensure that the time-step goes to zero as time is approaching the blow-up time. Hence, in this way, we avoid any possible instability, which may occur near blow-up time.

In this research, we use the explicit and linear implicit Euler finite difference schemes to compute the numerical blow-up solution and estimate the blow-up times for problem (1), where $p=3,4,5$, with $u_{0}(x)=100\left(x-x^{2}\right)$. In order to increase the order of numerical convergence and get more accurate results, a special time-steps formula, dependent on $p$, will be used with these schemes:

$k_{n}=\left\{\begin{array}{cc}\min \left(\frac{h^{2}}{3}, \frac{h^{\alpha}}{\left(\left\|U_{h}^{n}\right\|_{\infty}\right)^{p}}\right) & \text { for explicit Scheme } \\ \frac{h^{\alpha}}{\left(\left\|U_{h}^{n}\right\|_{\infty}\right)^{p}} & \text { for implicit scheme }\end{array}\right\}, n \geq 0, \alpha>0$

Moreover, the numerical simulations will be carried out to support the numerical findings and to confirm the known theoretical blow-up results. 


\section{FINITE DIFFERENCE SCHEMES}

In this section, we recall the semi discrete problem for problem (1) will be used eventually to derive the explicit and linear implicit Euler schemes.

Let $\mathrm{I}$ be a positive integer, and consider the grid $x_{i}=i h, 0 \leq i \leq I$, where $h=1 / I$. We can approximate the solution $u$ of problem (1) by the solution:

$U_{h}(\mathrm{t})=\left(U_{0}(t), U_{1}(t), \ldots U_{I}(t)\right)^{T}$.

of the following semidiscrete problem with using central finite difference operator of second order to replace the second space derivative:

$$
\left\{\begin{array}{l}
\frac{d}{d t} U_{i}-\frac{U_{i+1}-2 U_{i}+U_{i-1}}{h^{2}}=U_{i}^{p}, \quad 1 \leq i \leq I-1 \\
U_{0}(t)=U_{I}(t)=0 \\
U_{i}(0)=u_{0}\left(x_{i}\right), \quad 0 \leq i \leq I .
\end{array}\right\}
$$

Definition 2.1 [14]: Let $U_{h}$ be nonnegative solution of problem (2). We say that $U_{h}$ blow-up in finite time, if there exists $T_{h} \leq \infty$ such that:

$\left\|U_{h}(t)\right\|_{\infty}<\infty$, for $\mathrm{t} \in\left[0, T_{h}\right)$

$\left\|U_{h}(t)\right\|_{\infty} \rightarrow \infty$, as $\mathrm{t} \rightarrow T_{h}^{-}$

where $\left\|U_{h}(t)\right\|_{\infty}=\max _{0<i \leq I}\left|U_{i}(t)\right|$.

The next theorem shows that $\forall t \in(0, T)$, the solution of problem (2.4) approximate the solution of problem (2.1), as $h \rightarrow 0$.

Theorem 2.1 [14]:

Assume that $u \in c^{4,1}([0,1] \times[0, T])$, where $u$ is the solution of problem (1). Then for $h$ sufficiently small, problem (2) has a unique solution:

$U_{h} \in C^{1}\left([0, T], R^{I+1}\right)$ such that:

$$
\max _{0 \leq t \leq T}\left\|U_{h}(t)-u_{h}(t)\right\|_{\infty}=\mathrm{O}\left(h^{2}\right), h \rightarrow 0 .
$$

The next theorem shows that blow-up time $T_{h}$ of problem (2) converges to the blow-up time of problem (1).

\section{Theorem 2.2 [14]:}

Let $u \in c^{4,2}([0,1] \times[0, T))$ be a blow-up solution of problem (1) and $T$ is the blow-up time, such that:

$\lim _{t \rightarrow T} \int_{0}^{1} u(x, t) v(x) d x=\infty$,

where $v(x)$ is the solution of problem

$-v_{x x}=\lambda v, 0<x<1, v(0)=v(1)=0$,

Then for $h$ sufficiently small, $U_{h}$ blows up at $T_{h}<\infty$ and

$T_{h} \rightarrow T \quad$ as $\quad h \rightarrow 0$.

\subsection{Euler Explicit Schemes}

In order to derive the fully discrete explicit Euler finite difference equation to problem (1), we need to approximate the time derivative in problem (2) using the forward word finite difference formula as follows:

$\frac{U_{i}^{n+1}-U_{i}^{n}}{k}=\frac{U_{i+1}^{n}-2 U_{i}^{n}+U_{i-1}^{n}}{h^{2}}+U_{i}^{p}, 1 \leq i \leq I-1$
$U_{i}^{n+1}=\left(1-2 r_{h}^{n}\right) U_{i}^{n}+r_{h}^{n}\left(U_{i+1}^{n}+U_{1-1}^{n}\right)+U_{i}^{p}$,

where $U_{i}^{n}$ denotes the numerical of problem (1) at the point $\left(x_{i}, t_{n}\right)$,

$$
\begin{aligned}
& x_{i}=i h, \quad t_{n}=t_{n-1}+k_{n} ; \quad 1 \leq i \leq I-1, n=1,2, \ldots \ldots \\
& U_{h}^{n}=\left(U_{1}^{n}, U_{2}^{n} \ldots U_{I-1}^{n}\right)^{T}, \quad r_{h}^{n}=\frac{k_{n}}{h^{2}}
\end{aligned}
$$

It is well known that $\frac{k_{n}}{h^{2}} \leq 1$ is the stability condition of the explicit Euler scheme for heat equation, [8]. So that, to ensure and speed up the convergence, the time-steps will be chosen as follows:

$$
k_{n}=\min \left(\frac{h^{2}}{3}, \frac{h^{\alpha}}{\left(\left\|U_{h}^{n}\right\|_{\infty}\right)^{p}}\right) \quad, \alpha>0
$$

The discrete problem (3) can be written in a matrix form as follows: 
where $\quad H=\left[\begin{array}{cccc}-2 & 1 & & 0 \\ 1 & -2 & 1 & \\ & & \ddots & \\ 0 & & 1 & -2\end{array}\right]_{(m-1) \times(m-1)}^{n+1}=\left(I+r_{h}^{n} H\right) U_{h}^{n}+k_{n} F_{h}^{n}$,

\subsection{Euler Linear Implicit Scheme}

Secondly, we derive linear implicit Euler formula, in this case we replace the time derivative in problem (2) using the backward finite difference formula, as follows:

or

$$
\frac{U_{i}^{n+1}-U_{i}^{n}}{k_{n}}=\frac{U_{i+1}^{n+1}-2 U_{i}^{n+1}+U_{i-1}^{n+1}}{h^{2}}+\left(U^{n}\right)^{p}
$$

where

$$
\left(1+2 r_{h}^{n}\right) U_{i}^{n+1}-r_{h}^{n}\left(U_{i+1}^{n+1}+U_{i-1}^{n+1}\right)=U_{i}^{n}+U_{i}^{p}, 1 \leq i \leq I-1
$$

$$
k_{n}=\left(\frac{h^{\alpha}}{\left(\left\|U_{h}^{n}\right\|_{\infty}\right)^{p}}\right) \quad, \alpha>0
$$

The matrix form of linear Euler implicit method can be written as follows:

where $H$ takes the form as in (4).

$$
\left(I-r_{h}^{n} H\right) U_{h}^{n+1}=U_{h}^{n}+k_{n} F_{h}^{n}
$$

Remark 2.1: At each time level n, in order to find the approximate value of the vector $U_{h}^{n}$, we need to solve the linear system (5).

Definition 2.2 [15]: We say that the solution of the explicit (implicit) Euler scheme blows up in a finite time, if

1- $\left\|U_{h}^{n}\right\|_{\infty} \rightarrow \infty$ as $n \rightarrow \infty$

2- $T_{h}=\sum_{n=0}^{\infty} k_{n}$

where $T_{h}$ is called the numerical blow-up time of the discrete problem.

Remark 2.2: The numerical blow-up time of the discrete solution depends on space step $h$ and also on the choice of time steps $k_{n}$. In addition, it is well known that, for each fixed time interval $[0, T]$, explicit (implicit) Euler numerical schemes give approximate solutions with rate of convergence, $\mathrm{O}\left(k+h^{2}\right)$, where $k=\max _{n} k_{n},[14]$, while with this choice of time-steps,(4) and (6), we have a rate of convergence as follows:

$\mathrm{O}\left(h^{\alpha}\right), \quad$ as $h \rightarrow 0$, for $\alpha \leq 2$.

\section{NUMERICAL EXAMPLES}

In this section, we will use the two discrete finite equation derived in section two (explicit and Implicit Euler methods). Three numerical experiments will be considered:

Problem 1: $\left\{\begin{array}{ll}u_{t}=u_{x x}+u^{3} & , x \in(0,1), t>0 \\ u(x, t)=0, & , x=0,1 \\ u(x, 0)=100\left(x-x^{2}\right) & , x \in(0,1)\end{array}\right\}$

Problem 2: $\quad\left\{\begin{array}{ll}u_{t}=u_{x x}+u^{4} & , x \in(0,1), t>0 \\ u(x, t)=0, & , x=0,1 \\ u(x, 0)=100\left(x-x^{2}\right) & , x \in(0,1)\end{array}\right\}$

Problem 3: $\left\{\begin{array}{ll}u_{t}=u_{x x}+u^{5} & , x \in(0,1), t>0 \\ u(x, t)=0, & , x=0,1 \\ u(x, 0)=100\left(x-x^{2}\right) & , x \in(0,1)\end{array}\right\}$

3.1 The time-steps, Error Bounds and order of convergence

For explicit Euler scheme, the time step, will be taken as follows:

$k_{n}=\min \left(\frac{h^{2}}{3}, \frac{h^{\alpha}}{\left(\left\|U_{h}^{n}\right\|_{\infty}\right)^{p}}\right), n \geq 0$,

while, for linear implicit Euler scheme the time-steps will be taken as follows:

$k_{n}=\left(\frac{h^{\alpha}}{\left(\left\|U_{h}^{n}\right\|_{\infty}\right)^{p}}\right), n \geq 0$, 
where $\alpha$ is a fixed positive constant.

As mentioned before, with (6) the stability condition of the explicit Euler method $\left(2 h^{-1} k_{n} \leq 1\right)$ is satisfied. In fact, we have chosen this type of time-step in order to examine experimentally the rate of convergence for the numerical blow-up times with respect to the space-steps. Therefore, we have taken different choices of $\alpha$.

\subsection{Numerical Blow-up Time}

Since the analytical (exact) solutions to problems 1,2 and 3 with the associated initial condition are not known, we can only estimate numerically the blow-up times. As we will see later that, the numerical solution for problem 1, 2 and 3 do not exist for all $\in N$, because they become unbounded (too large) at some time level $\mathrm{n}$.

In this section, the numerical blow-up time is compute at the first time that $\left\|U_{n}^{m}\right\|_{\infty} \geq 10^{6}$, and the value $t_{n}=\sum_{n=0}^{m} k_{n}$ is taken as the blow- up time of the discrete problems, which also can be considered the numerical blow-up time of the differential equations in problems 1,2 , and 3 . Moreover, the error bonds between any two numerical blow-up times $T_{2 h}$ and $T_{h}$ are computed respectively with discretization parameters (space-steps) $2 h$ and $h$, is defined as follows:

$E_{h}=\left|T_{2 h}-T_{h}\right|$,

In order to estimate experimentally the order of accuracy of the numerical blow-up times, the order of convergence will be estimated using the formula [8]:

$S_{h}=\frac{\log \left(E_{2 h} / E_{h}\right)}{\log (2)}$

\subsection{Numerical Results}

The two schemes (Euler explicit and Euler implicit) will be used to compute the numerical solution for each problem (1, 2 and 3), for different values of the space-step, while, the time-step formulas, (4) and (6), will be considered with $\alpha=1,2$. All the computational codes are written in Matlab.

In the next tables, we present blow-up times, the errors bound and the order of convergence and the CPU time in second, and $m$ represents the number of iteration when numerical blow-up occurs.

In tables (1) and (2), we present the numerical results of problem one, using explicit Euler scheme with respect to $\alpha=1$ and 2 , respectively.

In tables (3) and (4), we present the numerical results of problem one, using implicit Euler scheme with respect to $\alpha=1$ and 2 , respectively.

In tables (5) and (6), we present the numerical results of problem two, using explicit Euler scheme with respect to $\alpha=1$ and 2 , respectively.

In tables (7) and (8), we present the numerical results of problem two, using implicit Euler scheme with respect to $\alpha=1$ and 2 , respectively.

In tables (9) and (10), we present the numerical results of problem three, using explicit Euler scheme with respect to $\alpha=1$ and 2 , respectively.

In tables (11) and (12), we present the numerical results of problem three, using implicit Euler scheme with respect to $\alpha=1$ and 2 , respectively.

Table 1-Problem 1, $p=3$, Explicit Euler scheme, $\alpha=1$

\begin{tabular}{|cccccc|}
\hline $\mathrm{h}$ & $m$ & $T_{h}$ & CPUT & $E_{h}$ & $S_{h}$ \\
\hline $1 / 20$ & 16 & $8.217323 \mathrm{e}^{-04}$ & 0.032616 & $\ldots \ldots \ldots \ldots \ldots$ & $\ldots \ldots \ldots \ldots$ \\
$1 / 40$ & 44 & $8.153862 \mathrm{e}^{-04}$ & 0.065445 & $0.063461 \mathrm{e}^{-04}$ & $\ldots \ldots \ldots$. \\
$1 / 80$ & 153 & $8.128780 \mathrm{e}^{-04}$ & 0.141904 & $0.025081 \mathrm{e}^{-04}$ & 1.339217 \\
$1 / 160$ & 586 & $8.119270 \mathrm{e}^{-04}$ & 0.301804 & $0.009510 \mathrm{e}^{-04}$ & 1.399135 \\
$1 / 320$ & 2315 & $8.115748 \mathrm{e}^{-04}$ & 0.794801 & $0.003522 \mathrm{e}^{-04}$ & 1.433050 \\
\hline
\end{tabular}

Table 2-Problem 1, $p=3$, Explicit Euler scheme, $\alpha=2$

\begin{tabular}{|cccccc|}
\hline $\mathrm{h}$ & $m$ & $T_{h}$ & $\mathrm{CPUT}$ & $E_{h}$ & $S_{h}$ \\
\hline $1 / 20$ & 48 & $8.117361 \mathrm{e}^{-04}$ & 0.242958 & $\ldots \ldots \ldots \ldots$ & $\ldots \ldots \ldots$ \\
$1 / 40$ & 237 & $8.114347 \mathrm{e}^{-04}$ & 0.402206 & $0.003014 \mathrm{e}^{-04}$ & $\ldots \ldots \ldots$ \\
$1 / 80$ & 1299 & $8.113593 \mathrm{e}^{-04}$ & 0.713649 & $0.000754 \mathrm{e}^{-04}$ & 1.999042 \\
$1 / 160$ & 1634 & $8.113404 \mathrm{e}^{-04}$ & 1.473871 & $0.000188 \mathrm{e}^{-04}$ & 1.996178 \\
$1 / 320$ & 2545 & $8.113356 \mathrm{e}^{-04}$ & 3.613842 & $0.000048 \mathrm{e}^{-04}$ & 1.977279 \\
\hline
\end{tabular}


Table 3-Problem 1, $p=3$, Implicit Euler scheme, $\alpha=1$

\begin{tabular}{|cccccc|}
\hline $\mathrm{h}$ & $m$ & $T_{h}$ & CPUT & $E_{h}$ & $S_{h}$ \\
\hline $1 / 20$ & 19 & $8.114953 \mathrm{e}^{-04}$ & 0.181253 & $\ldots \ldots \ldots \ldots$ & $\ldots \ldots \ldots \ldots$ \\
$1 / 40$ & 52 & $8.113332 \mathrm{e}^{-04}$ & 0.345534 & $0.001621 \mathrm{e}^{-04}$ & $\ldots \ldots \ldots$. \\
$1 / 80$ & 189 & $8.112719 \mathrm{e}^{-04}$ & 0.658324 & $0.000613 \mathrm{e}^{-04}$ & 1.402925 \\
$1 / 160$ & 620 & $8.112509 \mathrm{e}^{-04}$ & 2.058695 & $0.000210 \mathrm{e}^{-04}$ & 1.545497 \\
$1 / 320$ & 2762 & $8.112444 \mathrm{e}^{-04}$ & 5.151270 & $0.000064 \mathrm{e}^{-04}$ & 1.691877 \\
\hline
\end{tabular}

Table 4-Problem 1, $p=3$, Implicit Euler scheme, $\alpha=2$

\begin{tabular}{|cccccc|}
\hline $\mathrm{h}$ & $m$ & $T_{h}$ & CPUT & $E_{h}$ & $S_{h}$ \\
\hline $1 / 20$ & 50 & $8.115572 \mathrm{e}^{-04}$ & 0.314055 & $\ldots \ldots \ldots \ldots$ & $\ldots \ldots \ldots .$. \\
$1 / 40$ & 241 & $8.113178 \mathrm{e}^{-04}$ & 0.712546 & $0.002394 \mathrm{e}^{-04}$ & $\ldots \ldots \ldots$. \\
$1 / 80$ & 1314 & $8.112552 \mathrm{e}^{-04}$ & 1.337102 & $0.000625 \mathrm{e}^{-04}$ & 1.935188 \\
$1 / 160$ & 1675 & $8.112394 \mathrm{e}^{-04}$ & 4.003412 & $0.000158 \mathrm{e}^{-04}$ & 1.986238 \\
$1 / 320$ & 2690 & $8.112354 \mathrm{e}^{-04}$ & 18.222905 & $0.000040 \mathrm{e}^{-04}$ & 1.981852 \\
\hline
\end{tabular}

Table 5-Problem (2), $p=4$, Explicit Euler scheme, $\alpha=1$

\begin{tabular}{|cccccc|}
\hline $\mathrm{h}$ & $m$ & $T_{h}$ & CPUT & $E_{h}$ & $S_{h}$ \\
\hline $1 / 20$ & 5 & $2.705021 \mathrm{e}^{-05}$ & 0.173642 & $\ldots \ldots \ldots \ldots$ & $\ldots \ldots \ldots .$. \\
$1 / 40$ & 9 & $2.334890 \mathrm{e}^{-05}$ & 0.236839 & $0.370131 \mathrm{e}^{-05}$ & $\ldots \ldots \ldots$. \\
$1 / 80$ & 29 & $2.204093 \mathrm{e}^{-05}$ & 0.421795 & $0.130797 \mathrm{e}^{-05}$ & 1.500706 \\
$1 / 160$ & 156 & $2.161953 \mathrm{e}^{-05}$ & 0.760673 & $0.042140 \mathrm{e}^{-05}$ & 1.634067 \\
$1 / 320$ & 802 & $2.149072 \mathrm{e}^{-05}$ & 1.426539 & $0.012881 \mathrm{e}^{-05}$ & 1.709945 \\
\hline
\end{tabular}

Table 6-Problem (2), $p=4$, Explicit Euler scheme, $\alpha=2$

\begin{tabular}{|cccccc|}
\hline $\mathrm{h}$ & $m$ & $T_{h}$ & CPUT & $E_{h}$ & $S_{h}$ \\
\hline $1 / 20$ & 6 & $2.133134 \mathrm{e}^{-05}$ & 0.243383 & $\ldots \ldots \ldots$ & $\ldots \ldots \ldots$ \\
$1 / 40$ & 11 & $2.141119 \mathrm{e}^{-05}$ & 0.407437 & $0.007985 \mathrm{e}^{-05}$ & $\ldots \ldots \ldots$ \\
$1 / 80$ & 35 & $2.143078 \mathrm{e}^{-05}$ & 0.762645 & $0.001959 \mathrm{e}^{-05}$ & 2.027175 \\
$1 / 160$ & 166 & $2.143572 \mathrm{e}^{-05}$ & 1.433216 & $0.000494 \mathrm{e}^{-05}$ & 1.987534 \\
$1 / 320$ & 904 & $2.143697 \mathrm{e}^{-05}$ & 2.679134 & $0.000125 \mathrm{e}^{-05}$ & 1.982582 \\
\hline
\end{tabular}

Table 7-Problem (2), $p=4$, Implicit Euler scheme, $\alpha=1$

\begin{tabular}{|cccccc|}
\hline $\mathrm{h}$ & $m$ & $T_{h}$ & CPUT & $E_{h}$ & $S_{h}$ \\
\hline $1 / 20$ & 5 & $2.438780 \mathrm{e}^{-05}$ & 0.185578 & $\ldots \ldots \ldots$ & $\ldots \ldots \ldots \ldots$ \\
$1 / 40$ & 10 & $2.253194 \mathrm{e}^{-05}$ & 0.382259 & $0.185586 \mathrm{e}^{-05}$ & $\ldots \ldots \ldots$ \\
$1 / 80$ & 31 & $2.179634 \mathrm{e}^{-05}$ & 0.752232 & $0.073560 \mathrm{e}^{-05}$ & 1.335094 \\
$1 / 160$ & 160 & $2.154803 \mathrm{e}^{-05}$ & 2.230338 & $0.024831 \mathrm{e}^{-05}$ & 1.566779 \\
$1 / 320$ & 810 & $2.147023 \mathrm{e}^{-05}$ & 8.852154 & $0.007780 \mathrm{e}^{-05}$ & 1.674300 \\
\hline
\end{tabular}


Table 8-Problem (2), $p=4$, Implicit Euler scheme, $\alpha=2$

\begin{tabular}{|cccccc|}
\hline $\mathrm{h}$ & $m$ & $T_{h}$ & CPUT & $E_{h}$ & $S_{h}$ \\
\hline $1 / 20$ & 7 & $2.144663 \mathrm{e}^{-05}$ & 0.323161 & $\ldots \ldots \ldots$ & $\ldots \ldots \ldots$ \\
$1 / 40$ & 12 & $2.143999 \mathrm{e}^{-05}$ & 0.646921 & $0.000664 \mathrm{e}^{-05}$ & $\ldots \ldots \ldots$. \\
$1 / 80$ & 38 & $2.143798 \mathrm{e}^{-05}$ & 1.506066 & $0.000201 \mathrm{e}^{-05}$ & 1.723987 \\
$1 / 160$ & 170 & $2.143752 \mathrm{e}^{-05}$ & 4.381798 & $0.000046 \mathrm{e}^{-05}$ & 2.127489 \\
$1 / 320$ & 910 & $2.143742 \mathrm{e}^{-05}$ & 18.229119 & $0.000010 \mathrm{e}^{-05}$ & 2.201633 \\
\hline
\end{tabular}

Table 9 -roblem 3, $p=5$, Explicit Euler scheme, $\alpha=1$

\begin{tabular}{|cccccc|}
\hline $\mathrm{h}$ & $m$ & $T_{h}$ & CPUT & $E_{h}$ & $S_{h}$ \\
\hline $1 / 20$ & 4 & $6.908534 \mathrm{e}^{-07}$ & 0.150712 & $\ldots \ldots \ldots \ldots$ & $\ldots \ldots \ldots$. \\
$1 / 40$ & 8 & $6.659545 \mathrm{e}^{-07}$ & 0.250338 & $0.248989 \mathrm{e}^{-07}$ & $\ldots \ldots \ldots \ldots$ \\
$1 / 80$ & 29 & $6.528684 \mathrm{e}^{-07}$ & 0.448085 & $0.130860 \mathrm{e}^{-07}$ & 0.928046 \\
$1 / 160$ & 139 & $6.461718 \mathrm{e}^{-07}$ & 0.777276 & $0.066966 \mathrm{e}^{-07}$ & 0.966534 \\
$1 / 320$ & 780 & $6.427855 \mathrm{e}^{-07}$ & 1.387930 & $0.033863 \mathrm{e}^{-07}$ & 0.983719 \\
\hline
\end{tabular}

Table 10-Problem 3, $p=5$, Explicit Euler scheme, $\alpha=2$

\begin{tabular}{|cccccc|}
\hline $\mathrm{h}$ & $m$ & $T_{h}$ & $\mathrm{CPUT}$ & $E_{h}$ & $S_{h}$ \\
\hline $1 / 20$ & 5 & $6.430133 \mathrm{e}^{-07}$ & 0.254173 & $\ldots \ldots \ldots$ & $\ldots \ldots \ldots$ \\
$1 / 40$ & 10 & $6.420118 \mathrm{e}^{-07}$ & 0.411752 & $0.010014 \mathrm{e}^{-07}$ & $\ldots \ldots \ldots$ \\
$1 / 80$ & 31 & $6.422077 \mathrm{e}^{-07}$ & 0.729479 & $0.001958 \mathrm{e}^{-07}$ & 2.353973 \\
$1 / 160$ & 148 & $6.422571 \mathrm{e}^{-07}$ & 1.363684 & $0.000494 \mathrm{e}^{-07}$ & 1.987534 \\
$1 / 320$ & 803 & $6.422696 \mathrm{e}^{-07}$ & 2.733435 & $0.000125 \mathrm{e}^{-07}$ & 1.982582 \\
\hline
\end{tabular}

Table 11-Problem 3, $p=5$, Implicit Euler scheme, $\alpha=1$

\begin{tabular}{|cccccc|}
\hline $\mathrm{h}$ & $m$ & $T_{h}$ & CPUT & $E_{h}$ & $S_{h}$ \\
\hline $1 / 20$ & 4 & $6.481121 \mathrm{e}^{-07}$ & 0.181134 & $\ldots \ldots \ldots$ & $\ldots \ldots \ldots$. \\
$1 / 40$ & 9 & $6.447556 \mathrm{e}^{-07}$ & 0.371425 & $0.033565 \mathrm{e}^{-07}$ & $\ldots \ldots \ldots$ \\
$1 / 80$ & 30 & $6.433019 \mathrm{e}^{-07}$ & 0.771574 & $0.014537 \mathrm{e}^{-07}$ & 1.207228 \\
$1 / 160$ & 142 & $6.427204 \mathrm{e}^{-07}$ & 2.325200 & $0.005815 \mathrm{e}^{-07}$ & 1.321878 \\
$1 / 320$ & 798 & $6.424901 \mathrm{e}^{-07}$ & 8.597677 & $0.002302 \mathrm{e}^{-07}$ & 1.336264 \\
\hline
\end{tabular}

Table 12-Problem 3, $p=5$, Implicit Euler scheme, $\alpha=2$

\begin{tabular}{|cccccc|}
\hline $\mathrm{h}$ & $m$ & $T_{h}$ & CPUT & $E_{h}$ & $S_{h}$ \\
\hline $1 / 20$ & 5 & $6.422664 \mathrm{e}^{-07}$ & 0.321618 & $\ldots \ldots \ldots \ldots \ldots \ldots \ldots \ldots \ldots$ & $\ldots \ldots \ldots \ldots \ldots \ldots \ldots \ldots \ldots$ \\
$1 / 40$ & 10 & $6.421429 \mathrm{e}^{-07}$ & 0.665700 & $0.001235 \mathrm{e}^{-07}$ & $\ldots \ldots \ldots \ldots .$. \\
$1 / 80$ & 33 & $6.421120 \mathrm{e}^{-07}$ & 1.424917 & $0.000309 \mathrm{e}^{-07}$ & 1.998832 \\
$1 / 160$ & 151 & $6.421042 \mathrm{e}^{-07}$ & 4.642498 & $0.000078 \mathrm{e}^{-07}$ & 1.986060 \\
$1 / 320$ & 812 & $6.421023 \mathrm{e}^{-07}$ & 17.445631 & $0.000019 \mathrm{e}^{-07}$ & 2.037474 \\
\hline
\end{tabular}

\subsection{Numerical Simulations}

In this section, the numerical simulations are carried out to visualize the numerical graphs for the numerical blow-up solution of problems 1,2 and 3 obtained from using explicit and linear implicit Euler schemes, with $h=320$ and $\alpha=2$.

Figure-(1,2) present the discrete graph of the numerical solution of problem (1) obtained from using explicit and implicit schemes, respectively.

Figure- $(3,4)$ present the discrete graph of the numerical solution of problem (2) obtained from using explicit and implicit schemes, respectively.

Figure $(5,6)$ present the discrete graph of the numerical solution of problem (3) obtained from using explicit and implicit schemes, respectively. 


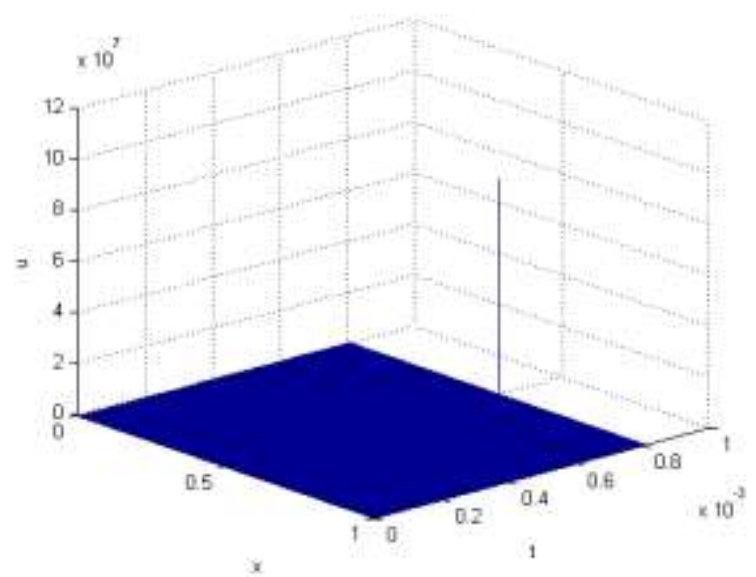

Figure 1-Problem (1), explicit scheme

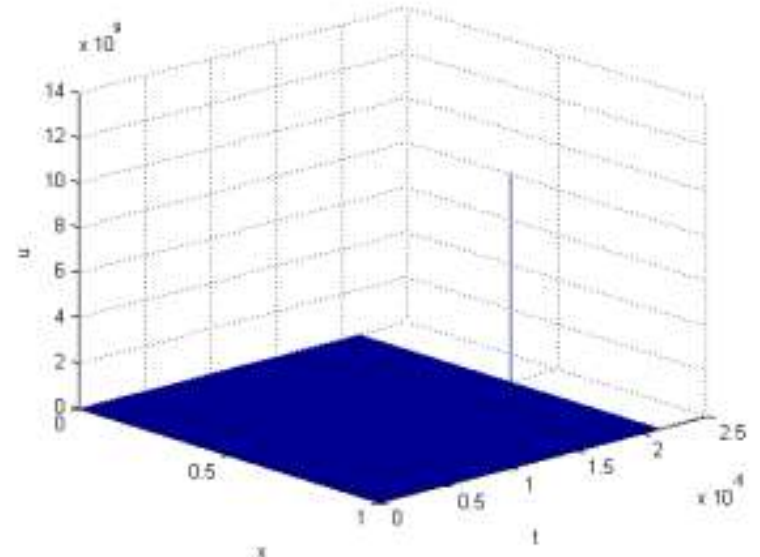

Figure 3-Problem (2), explicit scheme

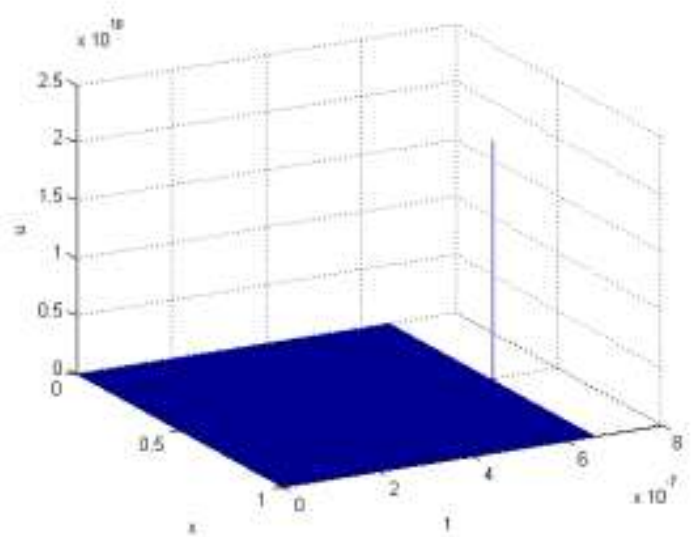

Figure 5-Problem (3), explicit scheme

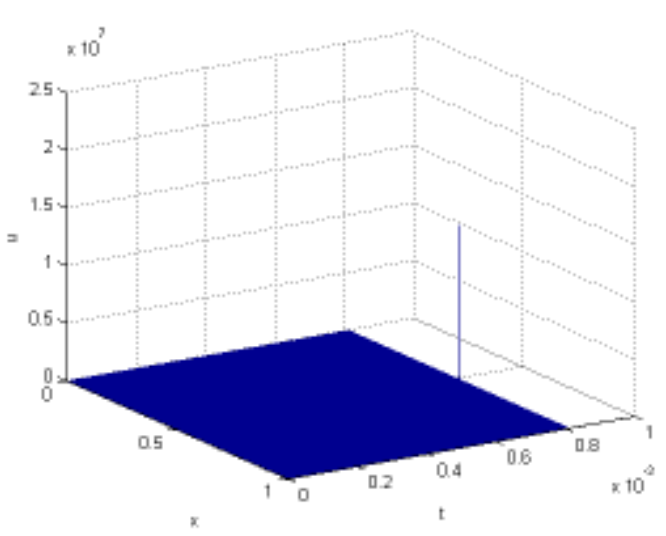

Figure 2-Problem (1), implicit scheme

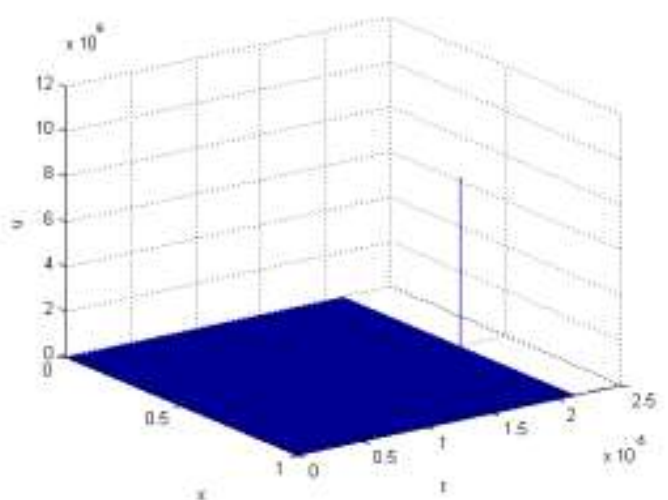

Figure 4-Problem (1), implicit scheme

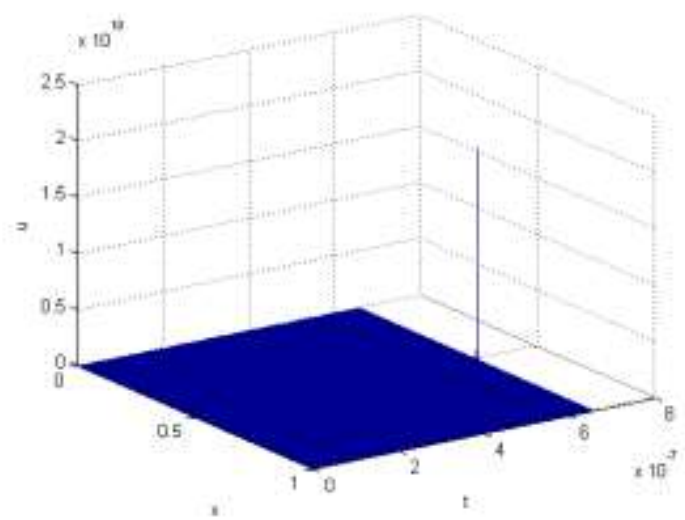

Figure 6-Problem (3), implicit scheme

\subsection{Analysis and Discussion}

From the numerical results in section 3 and 4, we can point out the following observations:

1. In each of problems 1,2 and 3 , the numerical blow-up can only accour at the center point $(x=$ 0.5 ), and that confirms the known theoretical blow-up results for semilinear heat equations, see [8].

2. The numerical blow-up times are decreasing as we increase the power of the nonlinear term in the semi-linear heat equation. In fact, this result can be proved theoretically using maximum principle, [20].

3. The errors bounds are decreasing as we refine the space-steps, which indicates that the sequence of numerical blow-up times is convergent, as the space-step goes to zero. 
4. The order of convergence of numerical blow-up time, $S_{h}$ is almost close to 2, for $\boldsymbol{\alpha}=2$, while $1 \leq S_{h}<2$, for $\boldsymbol{\alpha}=1$, as the space-step goes to zero, which indicates that with this choice of $k_{n}$, for $\alpha=2$, we have a rate of convergence:

$\mathrm{O}\left(h^{\alpha}\right)$, as $h \rightarrow 0$.

5. The numbers of iterations are increasing as we increase the value of $\boldsymbol{\alpha}$, or as we turn from the using explicit scheme to the implicit scheme.

6. CPU time is increasing, as we refine the grids with respect to space and time.

7. The numerical simulations show that the growth rate of blow-up solution for each of studied problems, arises from using explicit Euler scheme, is almost the same as that arises from using implicit Euler scheme.

\section{CONCLUSIONS}

In this research, we have proposed two algorithms for the numerical solution of semi-linear heat equations. The numerical blow-up solutions are computed for semi-linear heat equations with Dirichlet boundary conditions. Explicit and implicit Euler finite difference schemes with a special time-steps formula are presented and analyzed in order to solve the proposed problem and estimate the blow-up times. The numerical result obtained by the proposed methods is analyzed, simulated and presented in the form of tables and figures. Numerical examples show that the proposed methods are successfully implemented with good efficiency and high order of convergence.

\section{REFERENCES}

1. Zahire, S and Bülent K. 2013. Optimal boundary control for time-dependent diffusion-convectionreaction equations, International Journal of Mathematical Modelling and Numerical Optimisation, 4(3): $282-300$.

2. Itishree, N., Ajit., K. N. and Sudarsan, P. 2016. Implicit finite difference solution for the magnetohydro-dynamic unsteady free convective flow and heat transfer of a third-grade fluid past a porous vertical plate, International Journal of Mathematical Modelling and Numerical Optimisation, 7(1): 4-19.

3. Jameel, A. F., Saaban, A., and Zureigat, H. H. 2018. Numerical solution of second-order fuzzy nonlinear two-point boundary value problems using combination of finite difference and Newton's methods, Neural Computing and Applications, 30(10): 3167-3175.

4. Rasheed, M. A., Balasim, A. T. and Jameel, A. F. 2019. Some results for the vorticity transport equation by using A.D.I scheme, AIP Conference Proceedings, 2138, 030031, doi.org/10.1063/1.5121068

5. Rasheed, M. A., Laverty, S. , and Bannish, B. 2019. Numerical solutions of a linear agestructured population model, AIP Conference Proceedings, 2096, 020002, doi.org/10.1063/1.5097799

6. Lacey, A. A. 1998. Diffusion models with blow-up, Journal of Computational and Applied Mathematics, 97(1-2): 39-49.

7. Kaplan, S. 1963. On the growth of solutions of quasilinear parabolic Equations, Communications on Pure and Applied Mathematics, 16: 327-330.

8. Friedman, A. and McLeod, B. 1985. Blow-up of positive solutions of semi-linear heat equations, Indiana University Mathematics Journal, 34: 425-447.

9. Rasheed, M. A., Al-Dujaly, H. A. S. and Aldhlki, T. J. 2019. Blow-up rate estimates for a system of reaction-diffusion equations with gradient terms, International Journal of Mathematics and Mathematical Sciences, 2019: 1-7.

10. Han, Y. 2019. Blow-up at infinity of solutions to a semi-linear heat equation with logarithmic nonlinearity, Journal of Mathematical Analysis and Applications, 474(1): 513-517.

11. Rasheed, M. A. and Barghooth, L. J. 2019. Blow-up set and upper rate estimate for a semilinear heat equation, Journal of physics: Conf. Series 1294, 032013.

12. Rasheed, M. A. and Chlebik, M. 2020. Blow-up rate estimates and blow-up set for a system of two heat equations with coupled nonlinear neumann boundary conditions, Iraqi journal of Science, 61(1): $147-152$.

13. Friedman, A. 1964. Partial Differential Equations of Parabolic Type, Prentic-Hall, Englewood Cliffs, N.J. 
14. Abia, L.M., Lopez-Marcos, J.C and Martinez, J. 1995. Blow-up for semidiscretizations of reaction-diffusion equations, Applied Numerical Mathematics, 20: 145-156.

15. Abia, L.M., Lopez-Marcos, J.C and Martinez, J. 2001. The Euler method in the numerical integration of reaction-diffusion problems with blow-up, Applied Numerical Mathematics, 38: 287-313.

16. Chang, C. F., Wu, Y. C and Cho, C. H. 2015. Blow-up numerical solutions for a convective reaction-diffusion equation, Journal of Numerical Mathematics, 23(2): 117-136.

17. Cho, C. H. 2016. A numerical algorithm for blow-up problems revisited, Numerical Algorithms, 75(3): 675-697.

18. Polyanin, A. D. and Shingareva, I. K. 2018. Nonlinear problems with blow-up solutions: Numerical integration based on differential and nonlocal transformations, and differential constraints, Applied Mathematics and Computation, 336: 107-137.

19. Polyanin, A. D. and Shingareva, I. K. 2019. Nonlinear blow-up problems for systems of ODEs and PDEs: Non-local transformations, numerical and exact solutions, International Journal of Non-Linear Mechanics, 111: 28-41.

20. Quittner, P. and Souplet, Ph. 2007. Superlinear Parabolic Problems. Blow-up, Global Existence and Steady States, Birkhuser Advanced Texts, Birkhuser, Basel. 